\title{
Simulation Analysis of Computer-Controlled Pressurization for Mixture Ratio Control
}

\author{
Leslie A. Alexander and Karen Bishop-Behel \\ NASA Marshall Space Flight Center Code NP-40, Alabama 35812 \\ Michael P.J. Benfield \\ SAIC, Huntsville, Alabama 35806 \\ Anthony Kelley \\ NASA Marshall Space Flight Center Code EV-23, Alabama 35812
}

\author{
Gordon R. Woodcock, Consultant grw33@comcast.net \\ Gray Research, Inc. 655 Discovery Drive, Suite 300, Huntsville, Alabama 35806
}

\begin{abstract}
A procedural code $(\mathrm{C}++)$ simulation was developed to investigate potentials for mixture ratio control of pressure-fed spacecraft rocket propulsion systems by measuring propellant flows, tank liquid quantities, or both, and using feedback from these measurements to adjust propellant tank pressures to set the correct operating mixture ratio for minimum propellant residuals. The pressurization system eliminated mechanical regulators in favor of a computer-controlled, servodriven throttling valve. We found that a quasi-steady-state simulation (pressure and flow transients in the pressurization systems resulting from changes in flow control valve position are ignored) is adequate for this purpose. Monte-Carlo methods are used to obtain simulated statistics on propellant depletion. Mixture ratio control algorithms based on proportional-integral-differential (PID) controller methods were developed. These algorithms actually set target tank pressures; the tank pressures are controlled by another PID controller. Simulation indicates this approach can provide reductions in residual propellants.
\end{abstract}

\section{Introduction and Purpose}

One of the least reliable components in a pressure-fed rocket propulsion system is the mechanical regulator that controls tank pressurization. In addition to reliability issues, regulators are difficult to set, and uncertainties are associated with the delivered pressure. Current spacecraft practice uses separate regulators for the oxidizer and fuel to prevent accidental mixing of propellants in the pressurization gas system during long planetary coast periods. This introduces an additional problem: differences in the output pressures of the two regulators can aggravate operating mixture ratio differences and increase the residual propellant component of inert mass. Allowance for residual propellant contribution to inert mass must be enough that the probability of depletion of propellant prior to mission completion is very small.

The reported investigation was undertaken to determine the performance potential for active mixture ratio control systems for representative planetary spacecraft, thus to assess whether this technology is a promising area for technology advancement investment. Most if not all current spacecraft propulsion systems are pressure-fed, and this investigation was limited to pressure-fed systems.

Most current pump-fed engines are capable of changing mixture ratio over a modest range, and some launch vehicles use this capability for mixture ratio control. The main need for launch vehicle applications is better tank liquid quantity and flow measurement instruments. 
A note on units: We have taken the liberty of using SI units for all parameters in this paper except pressures. Most propulsion analysts are familiar with system pressure characteristics quoted in psia, so we follow that practice; $\mathrm{kPa}$ values are given in parentheses in some cases. Simulation calculations all used SI units.

\section{Initial Simulations}

Simulations of tank pressure control began a few years ago at Space America, during tests of a pressure-fed LOX-kerosene engine. Test stand experience with propellant pressurization regulators was less than satisfactory. An initial system using on-off solenoid valves was targeted. The method was simulated prior to implementation, to determine practicality and develop controller algorithms. A proportional-differential controller was used, with input from tank pressure transducers and the output digitized to an on/off time ratio.

The test stand computer control cycle was 50 milliseconds. The valves responded adequately, but the minimum pressurant flow bit (i.e. the minimum amount of gas delivered by one minimum-duration valve on-off cycle), with helium bottles at full pressure and minimum ullage in the tanks, led to pressure variance in ullage pressure about 5 psi. However, once propellant flow started, regulation was more accurate and repeatable than mechanical regulators.

This experience led to a concept using multi-turn throttling valves driven by "go-to" digital servo steppers. It was simulated, but never implemented due to lack of funds. That simulation was the starting point for the present effort.

\section{Mixture Ratio Control Approaches}

When separate active controllers are used for the two propellant tanks, the opportunity exists to adjust tank pressures to control the system operating mixture ratio. Variance in operating mixture ratio due to uncertainties in pressures and pressure drops are only a few percent. Tank pressure adjustments to control these are also only a few percent. If these variances are not controlled, residual propellant due to one propellant depleting sooner than the other is also only a few percent of loaded propellant, but in a typical propulsion system, that represents a significant fraction of propulsion system inert mass.

In order to effect propellant simultaneous depletion by controlling mixture ratio, accurate measurements are necessary. We considered three operating scenarios:

1. Amounts of propellants loaded are precisely known, for example, because the propellants are storable and are loaded in a propellant servicing facility where the spacecraft is weighed during loading. Flow measurement devices (flowmeters) are available for measuring propellant flows during system operation.

2. Amounts of propellants loaded and/or available are not precisely known, for example, because they are loaded on the pad. Also, one or both propellants may be cryogenic and some venting of boiloff may have occurred during a coast period prior to propulsion system use. Accurate measurement of liquid quantity in each tank is necessary. Liquid quantity measurements, either continuous or periodic (these cases are different!), are to be used for mixture ratio control.

3. As in case 2, propellants available are not accurately known. Liquid quantity measurements and flow measurements are to be used.

\section{Simulation Target}

The system simulated was a conventional spacecraft propulsion system. The schematic used for the simulation is shown in Figure 1. The figure shows half of the propulsion system, i.e. the oxidizer side. The fuel side is identical. The heat exchanger is optional and can be left out of the simulation. The purpose of simulating a heat exchanger was to evaluate the benefits of storing the helium pressurant cold 
(to reduce volume and mass of the helium tanks) and warming it before it enters the propellant tank. Cavitating venturis are also an optional feature. These are used to make a system insensitive to pressure drops downstream of the cavitating venturis. These devices when operating properly, i.e. cavitating, exhibit mass flow that is a function only of the upstream pressure. Downstream pressures adjust to the flow set by the cavitating venturis.

Typical systems operate with tank pressures 250 to 300 psia (1720 to $2070 \mathrm{kPa}$ ), combustion chamber pressures about $140 \mathrm{psia}(965 \mathrm{kPa})$, thruster injector pressure drop about $110 \mathrm{psia}(760 \mathrm{kPa})$, and cavitating venturi pressure drop (if used) about $35 \mathrm{psi}(240 \mathrm{kPa})$. Other propellant flow pressure drops are small.

Helium tank initial pressures are typically 4500 psia $(31 \mathrm{MPa})$, and at the end of propellant expulsion about $450 \mathrm{psia}(3.1 \mathrm{MPa})$.

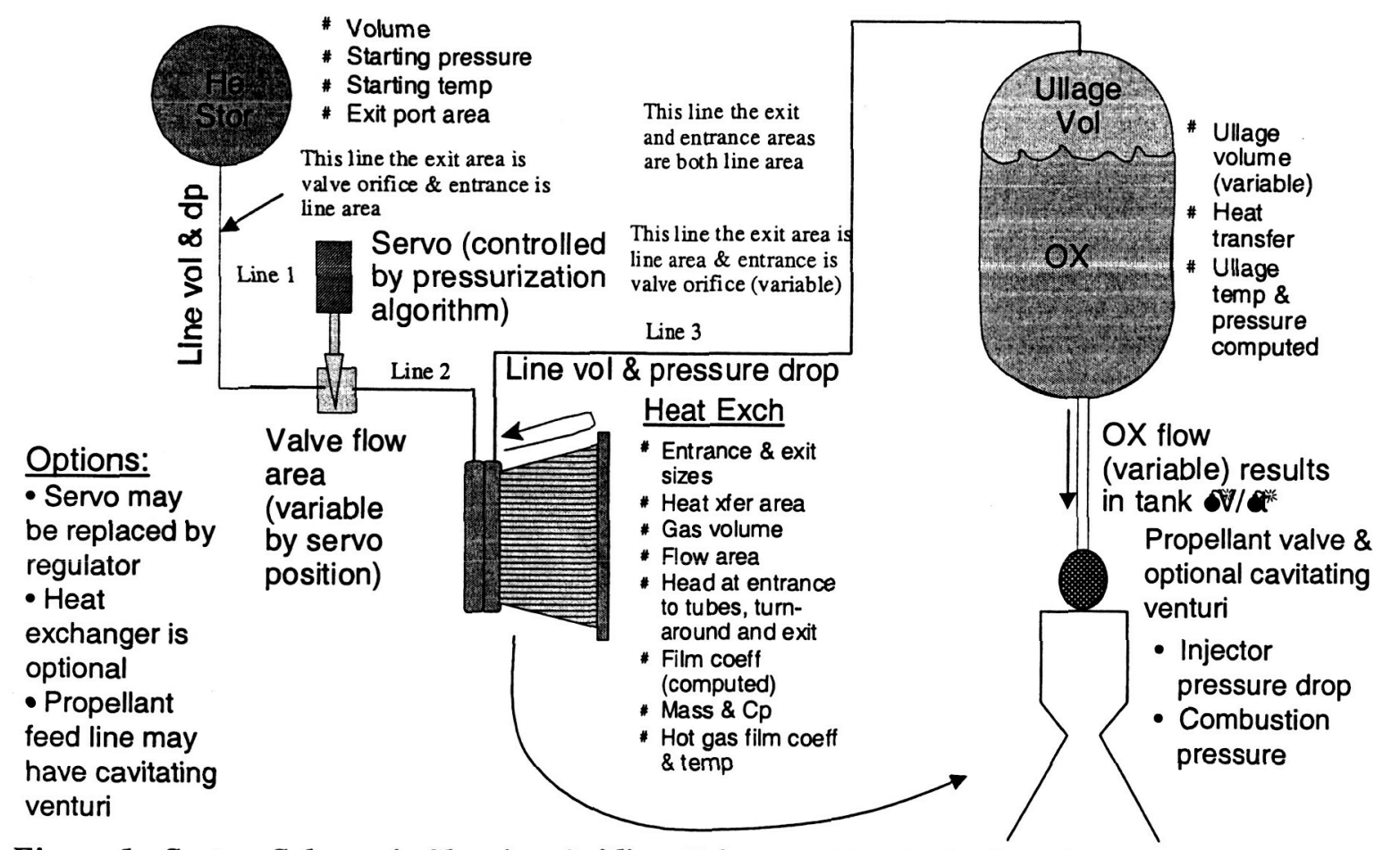

Figure 1: System Schematic Showing Oxidizer Side (Fuel Side is the Same)

The simulation considered the following pressurization system segments: (1) Source tank, (2) Line 1, from the tank to the control valve, (3) control valve, (4) Line 2, from the control valve to the heat exchanger, (5) Heat exchanger, (6) Line 3, from heat exchanger to propellant tank, (7) Propellant tank ullage, which varies in volume as propellant is expelled, (8) propellant tank heat capacity and heat exchange with the pressurant gas in the ullage volume (heat exchange with the propellant is also estimated, but propellant evaporation into the ullage volume was not simulated; this has essentially no effect on results for storable propellants that were the subject of this study), (9) liquid feed system pressure drop, (10) injector pressure drop, and (11) thruster chamber pressure. The latter was modeled as proportional to total propellant flow.

The controller simulated was assumed to be a throttling valve such as a needle valve, with roughly linear characteristics over the control range of travel. The valve is driven by a digital servo with rotary motion. We assumed valve travel of three complete rotations and a servo capable of 1-degree steps. This gave 1080 steps total, which we rounded to 1000 . The control algorithms do not depend on any particular valve calibration. 


\section{Simulation Methods}

Dynamic Simulation - We refer to dynamic simulation as a method that attempts to keep track of changing pressures, pressure drops, temperatures, and mass flows in and out as a function of time and controller settings. Mass flows in and out of each segment of the system are in general not equal. The first attempt at dynamic simulation tried to evaluate flow from the source tank through the system. Flow at the control valve was calculated first (it's usually choked), and calculations were then propagated sequentially upstream and downstream. This method was not satisfactory as it exhibited calculation instabilities and did not match conditions at the interfaces between system segments. Typical results are shown in Figure 2. The left-hand plots are pressures, the upper right is temperatures, and the lower right is flows. The upper left shows instabilities with a large time step. At the lower right, the instabilities are reduced by a much smaller time step. The stair-step shape of the curve results from stepwise opening of the control valve. The lower right shows that flows do not converge to a common value as they should. Interface conditions are not matched.
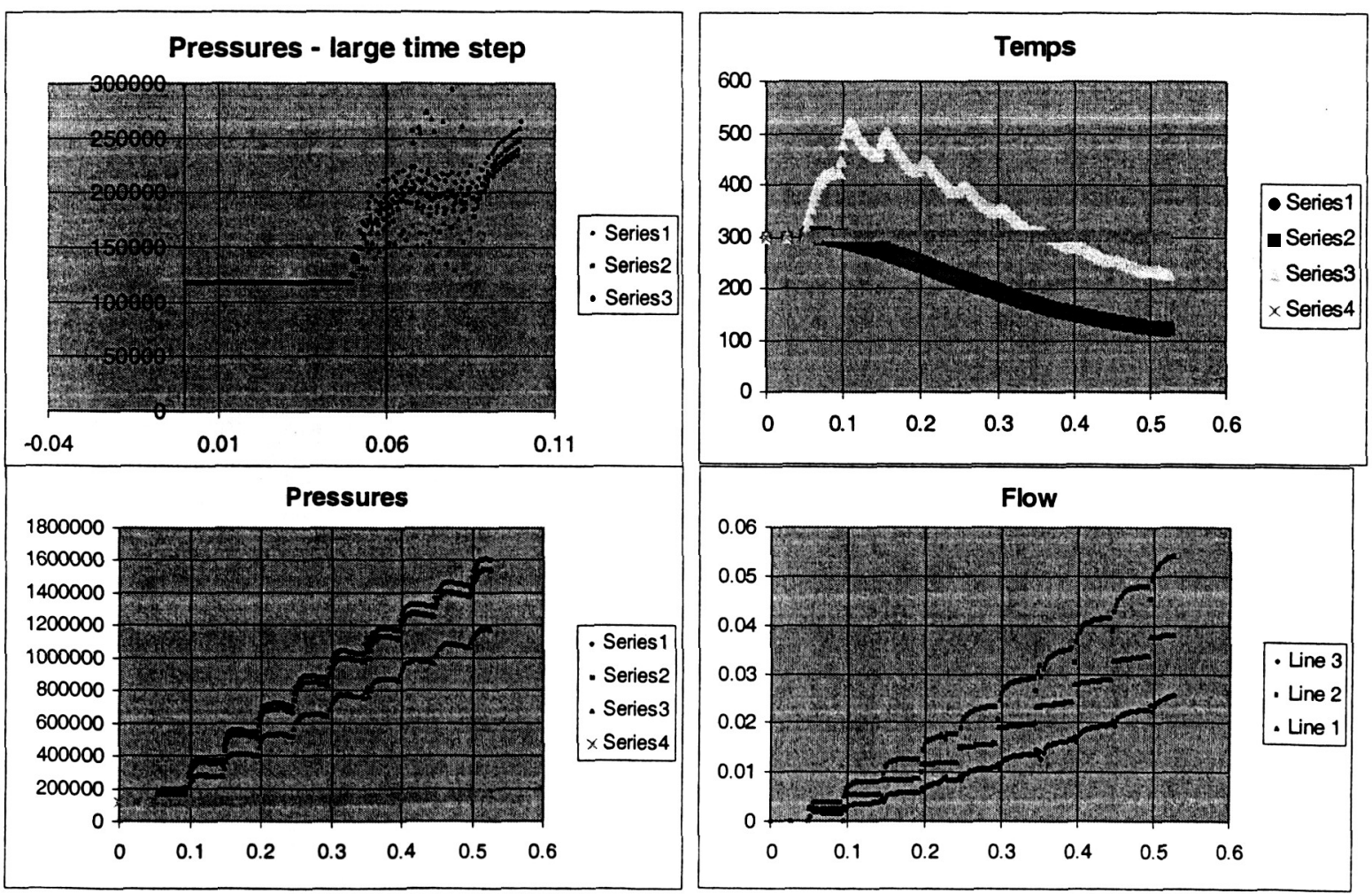

Large time step $500 \mu \mathrm{sec}$ upper left, smaller time step $50 \mu \mathrm{sec}$ stabilizes but flow results don't make sense

Figure 2: Results of Initial Simulation Approach

The second approach used a simultaneous solution based on first-order time derivatives and a Taylor's expansion. In this way, all the interactions among variables were solved jointly. If one considers, for example, the outflow from the storage tank: it is determined by the (small) pressure drop from the storage tank into Line 1:

$$
m=C p A / \operatorname{sqrt}(R T)
$$


where $\mathrm{C}$ is the flow coefficient, and $\mathrm{p}$ and $\mathrm{T}$ are upstream values. $\mathrm{R}$ is the gas constant, and $\mathrm{A}$ is flow area. The variables are $\mathrm{C}, \mathrm{p}$, and $\mathrm{T}$. The Taylor's expansion is

$$
\delta m=(\square m / \square C) \delta C+(\square m / \square p) \delta p+(\square m / \square T) \delta T
$$

Analogous expressions are written for all of the variables, and the partial derivatives are calculated. (They may be calculated from analytical expressions or derived in a computer program from the expressions for the variables by numerical differentiation; we chose to use analytical expressions.) The Taylor's expansion expressions are then placed in a linear algebraic matrix as illustrated in Figure 3 . The matrix is inverted to obtain a simultaneous solution for all the changes ( $\delta$ 's) in parameters, for a small time step. The process is repeated as a numerical integration. The test matrix shown in Figure 3 simulated the helium storage tank, Line 1, and the flow control valve. (Items in red were colored to aid in correcting errors.) "T" items are Taylor's expansions too lengthy to write into the matrix diagram

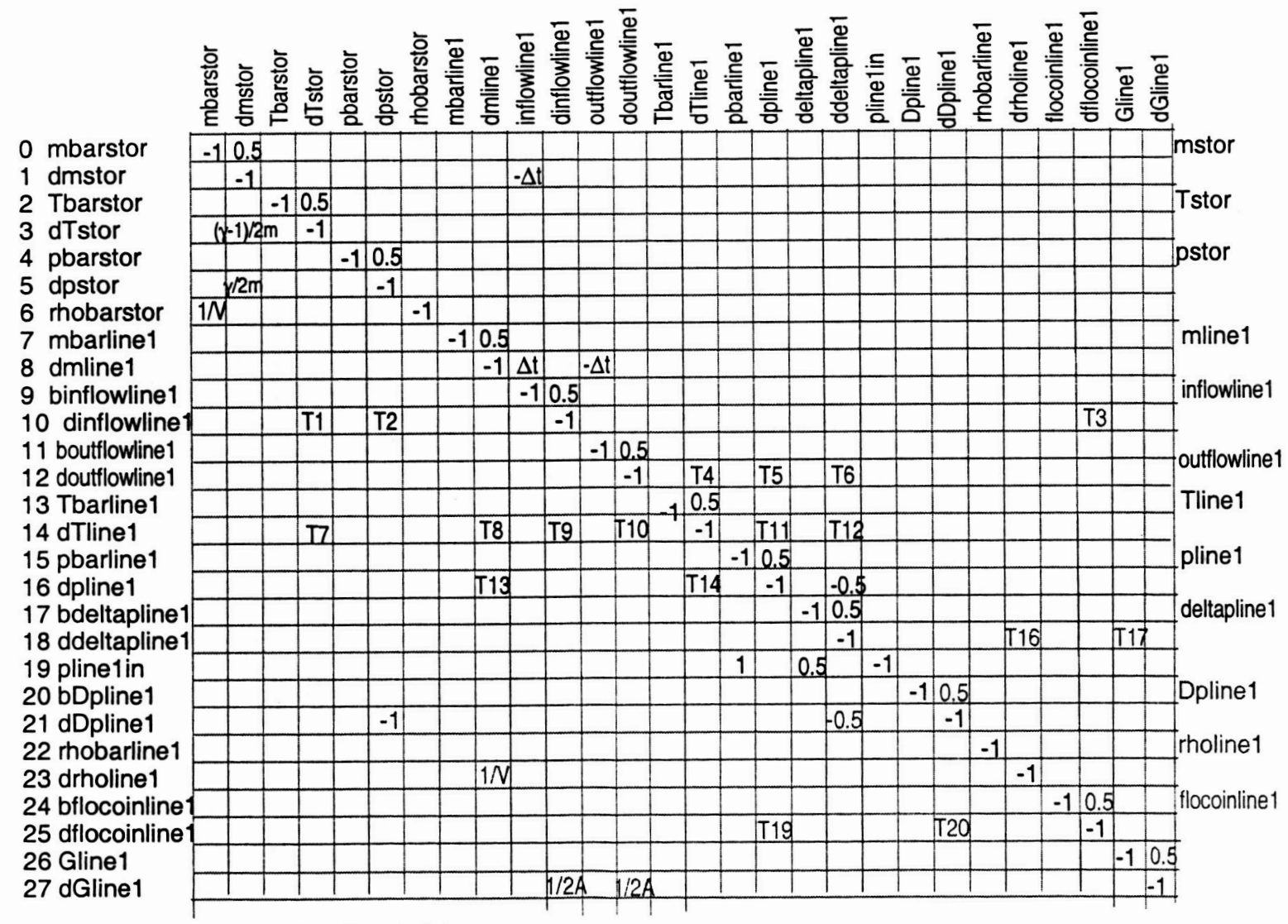

Figure 3: Test Matrix for Partial Simulation

Typical test results are shown in Figure 4, and showed (a) that the method works, i.e. initial values of parameters change to a steady-state in which flows and pressures are in balance; mass changes in Line 1 go to zero as steady state is reached; (b) the time to reach steady state is very short, typically 10 microseconds. Time steps about 1 microsecond were needed. In the simulation, the cycle time between helium control valve steps was $50 \mathrm{~ms}(50,000 \mu \mathrm{s})$. Therefore, we decided that simulating transients in the helium pressurant delivery system was unnecessary as well as impractical. A quasi-steady state solution 
method was adopted, in which it is assumed that helium flow is set by the control valve and is the same at any particular instant everywhere in the helium delivery system.

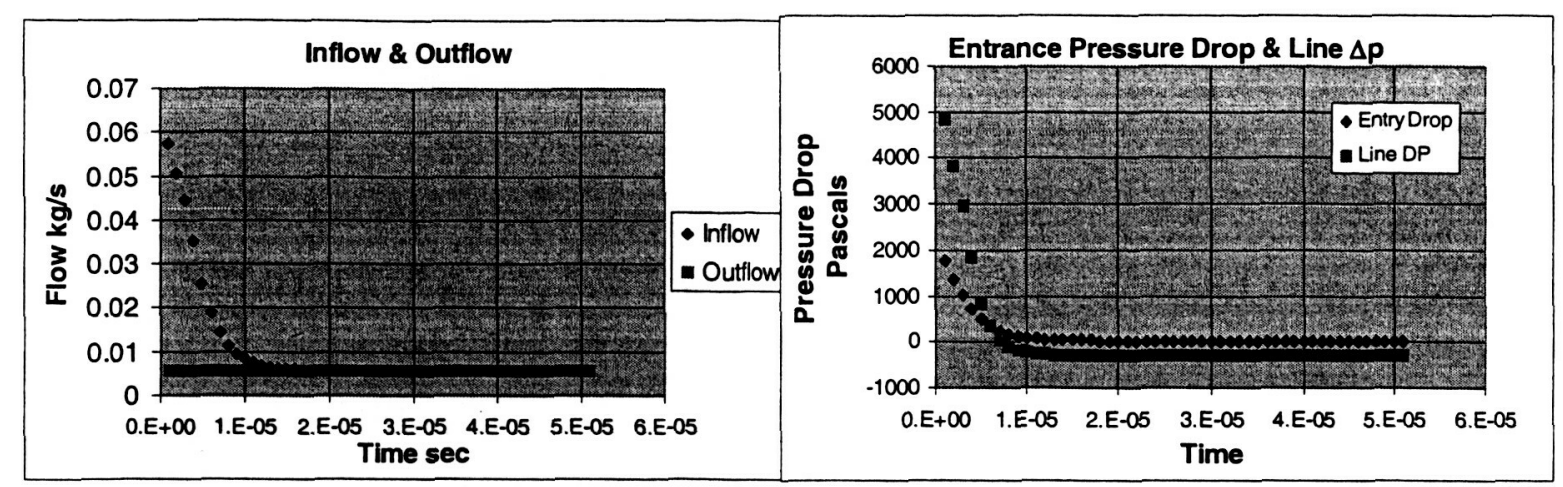

- Shows that the micro formulation approach works

- Pressures adjust until inflow = outflow

- Time constant is very short $\sim 10$ microseconds

- Don't need such fine resolution

- Line DP slightly negative is a consequence of finite integration step

- Easily correctable

Figure 4: Typical Results of Dynamic Simulation

Quasi-Steady-State Simulation - Flow is set by the control valve position. A pressure iteration is needed for the pressure drop through the control valve, which normally will be choked. The flow in Line 1 is always subsonic. The flow in Line 2 may be partially supersonic and shock down to subsonic flow. Constraints are that the static pressure at Line 3 exit into the propellant tank must be equal to the current ullage pressure (assuming the flow here is subsonic). Pressure downstream of the valve in Line 2 is iterated until this constraint is satisfied. Simulation time steps were half the assumed control cycle time, i.e. $25 \mathrm{~ms}$. Figure 5 shows an early test of this simulation, in which LOX tank ullage pressurization is simulated. The pressurization algorithm steps the valve open in discrete steps until the ullage pressure is about half the target value; then begins stepping the valve closed. When the ullage pressure reaches $90 \%$ of the target value, control authority is transferred to the run pressure control algorithm.

\section{Pressure Control Algorithms}

Two pressure control algorithms were tried: a predictor-corrector and a proportional-differential (PD) controller. The predictor-corrector attempted to determine the valve position that would result in the desired ullage pressure as a quasi-steady-state, based on measured values of current pressures, valve position, and rates of change of pressures. It then drove the valve to that position. The PD controller calculated a value based on gains applied to the pressure error and the rate of change of pressure. This value was converted to a valve step increment (amount of rotational motion), which was then executed. The valve step allowed for a single control cycle was limited to a maximum value to avoid excessive valve motion. Both worked; the PD controller was simpler and a little more stable, with less valve dithering. 

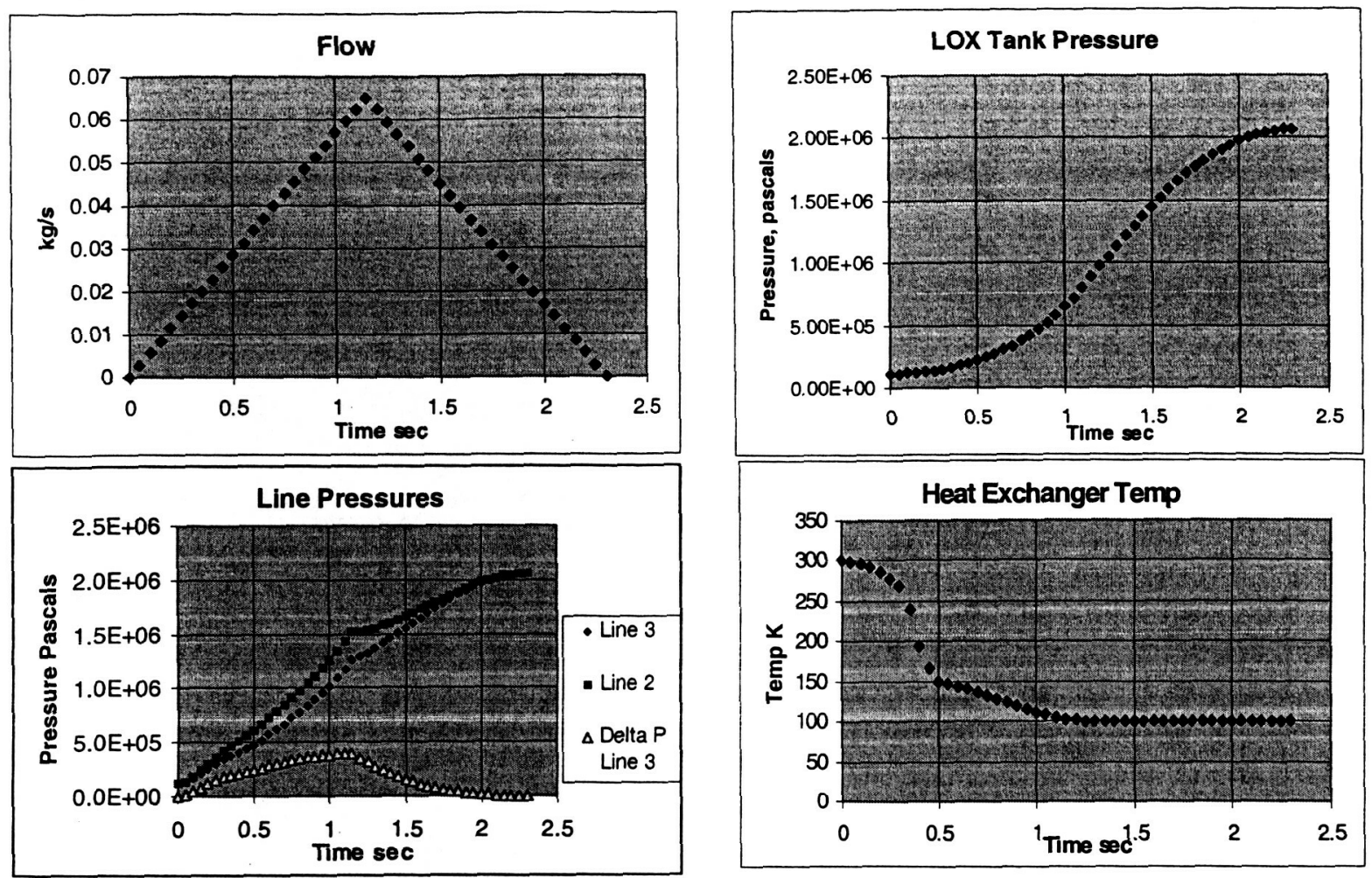

\section{Pressurization of LOX tank appears properly simulated}

\section{Figure 5: Early Test of Quasi-Steady-State Simulation}

\section{Mixture Ratio Control Measurement Methods}

Mixture ratio control feedback may be provided by propellant flowmeters, by tank quantity liquid measurement, or by both. The NASA Marshall Space Flight Center is performing R\&D on new technology for these purposes. For an active control system to exceed the performance of simple calibration requires that the measurements exhibit good accuracy. Historically, flow and quantity measurement methods have not offered enough accuracy to give an expectation of significant performance improvement.

A balanced flowmeter ${ }^{1}$ is being developed to measure liquid mass and volume flow. This is a pressure-drop device and does not have a rotor in the flow that could break up or otherwise fail in a hazardous manner. Two approaches are being taken to propellant tank liquid quantity measurement ${ }^{2,3}$; one is a variation on the PVT method and the other is an optical device. Measurements during thrusting operations, when vehicle acceleration settles the propellants, could also rely on point liquid sensors. As noted below, a number of sensors would be required.

\section{Mixture Ratio Control Algorithms}

1. Loaded propellant quantities accurately known: This is a classical proportional, integral, differential (PID) controller problem. The error signal is difference between the current run mixture ratio as determined by flowmeters, and the known loaded mixture ratio. A PID controller applies a gain to the error signal and adjusts the tank "set" pressures ["Set" pressure is the commanded pressure input to the tank pressure controller.) based on the gain. The integrator in the algorithm accumulates the error and 
applies a second gain. This causes the controller to correct for the small error the proportional factor must have to generate a corrective signal as well as prior accumulated error. The differential part of the controller applies negative feedback based on rate of change of error and stabilizes the controller.

2. Current propellant quantities not accurately known (as in cases of boiloff, or use of one propellant as a monopropellant for attitude control): There are two sub-cases, $2 \mathrm{a}$ and $2 \mathrm{~b}$. In $2 \mathrm{a}$, tank liquid quantity measurement is continuous or nearly so, and errors in the measurement do not vary in a random manner; that is, errors are systematic. In this case, an error signal can be derived from the rate of change in tank quantities mixture ratio. Driving this rate of change to zero means the tank quantities mixture ratio stays constant and both tanks will deplete simultaneously. In $2 b$, tank liquid quantity measurements exhibit errors that fluctuate in a random manner. This causes the $2 \mathrm{a}$ algorithm to become unstable because the differential part of the controller sees these random variations as large rates of change in tank mixture ratio, and outputs unacceptable fluctuations in tank "set" pressures.

In the $2 \mathrm{~b}$ case it is necessary to introduce some statistical smoothing to obtain a satisfactory algorithm. One way to do this is illustrated in Figure 6. Consecutive measurements are collected in circular buffers. Once enough are collected, regression lines are computed for rate of depletion of each propellant. These are extrapolated to zero propellant, which is the estimated depletion time. The difference in depletion times is the error signal. The controller attempts to drive this error to zero.

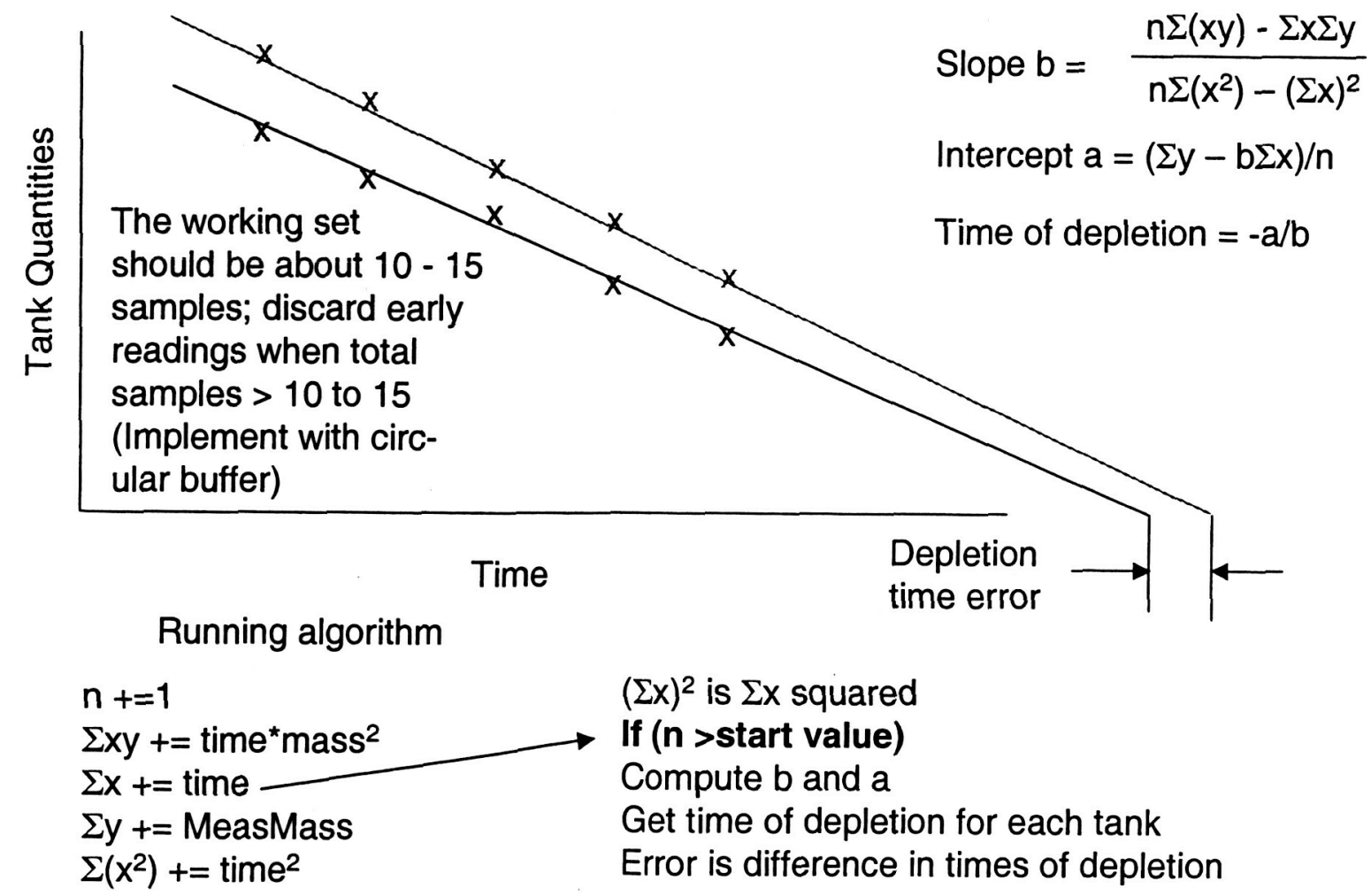

Figure 6: Regression Method for Using Tank Liquid Quantity Measurements

3. This one works the same as the first one, except that current measured tank liquid quantities are used instead of initial values. Flowmeter readings provide the current run mixture ratio, and the difference between tank and run mixture ratios is the error signal. The continuing measurements of tank mixture ratio provide the integration function, so an integrator is not needed in the controller. If tank 
liquid quantity measurements exhibit random fluctuations, current measured tank liquid quantities should be estimated from regression lines rather than from single measurements.

\section{Monte-Carlo Simulations}

Monte-Carlo simulation enables estimates of the statistics of the output of a system based on predicted random fluctuations of the inputs. The method is conceptually simple: a sequence of random numbers is generated, usually varying from 0 to 1 (with median 0.5 ). The probability of occurrence of a random number within any particular range, say, 0.01 to 0.02 , is equal to the probability of occurrence within any other equal range, say, 0.51 to 0.52 , in the interval 0,1 . In other words, the output of the random number generator is a "flat" (uniform) distribution. By virtue of the central limit theorem, random outputs of complicated physical systems vary according to the normal (Gaussian) distribution. The variances of inputs need to be converted from a uniform distribution to a normal distribution; therefore the random numbers in the sequence must be converted to a normal distribution. In this study we used a curve fit.

Figure 7 shows the use of the normal distribution integral curve to obtain the equivalent variance in standard deviations (sigmas) from the output of the random number generator. The mean of the uniform distribution is 0.5. As an example, the deviation from the mean of the vertical arrow is about 0.25 which corresponds to 0.68 sigma from the mean. If the random number generator outputs a value 0.75 , the variation in sigmas for the simulation input is 0.68 , and the 1-sigma value is multiplied by 0.68 . Similarly, if the random number output is 0.1 , the simulation input is varied by -1.2 sigma.

Variances were applied to inputs such as helium and propellant line pressure drops, thruster injector pressure drop, measurements of tank pressure, flowmeter measurements, and heat transfer in those cases where a heat exchanger was used. Early tests of the complete simulation, during which controller algorithms were developed, did not apply random errors to flowmeters and tank liquid quantity measurements. Later simulations included measurement errors.

During preparation of this paper, a better method of generating normally distributed random numbers was found. ${ }^{4}$ This uses a Box-Muller

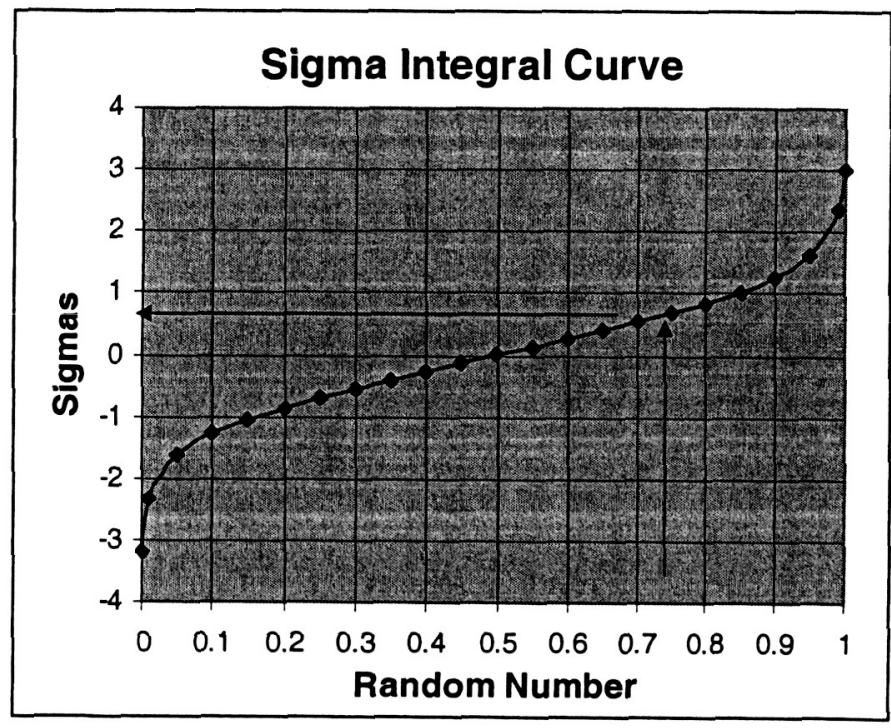

The random number obtained from the generator is input to this curve to get the number of sigmas it represents. The data base error value was taken as 1 sigma, and that value was multiplied by the number of sigmas to get the varied value. The generator is queried for each value from the data base, so the generator is queried almost 40 times for each simulation run.

\section{Figure 7: Use of Curve Fit to Obtain Number of Sigmas} transform. The method directly transforms a uniform distribution to a normal distribution as summarized in Figure 8. Uniformly distributed random numbers are generated in pairs, and from these a pair of normally distributed numbers is generated. As noted in the Figure, this only requires two lines of code (three counting the extra call of the uniform random number generator. A preliminary test is shown in the Figure. Statistical tests on the normal distribution were not performed. 


$$
\begin{aligned}
& z_{1}=\sqrt{-2 \ln x_{1}} \cos \left(2 \pi x_{2}\right) \\
& z_{2}=\sqrt{-2 \ln x_{1}} \sin \left(2 \pi x_{2}\right) .
\end{aligned}
$$

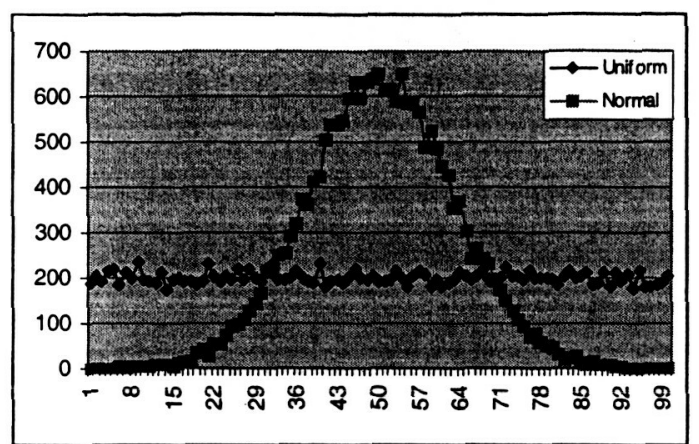

Figure 8: Box-Muller Transform Method

$$
\begin{aligned}
& \operatorname{ranx1}=\operatorname{rangen}(\& \operatorname{seed} 1, \& \operatorname{seed} 2, \& \text { seed3 }) ; \\
& \operatorname{ranx2}=\operatorname{rangen}(\& \operatorname{seed} 1, \& \operatorname{seed} 2, \& \operatorname{seed} 3) ; \\
& \operatorname{ranz1}=\operatorname{sqrt}\left(-2^{*} \log (\operatorname{ran} \times 1)\right)^{*} \cos \left(2^{*} \mathrm{pi}^{\star} \operatorname{ran} \times 2\right) ; \\
& \operatorname{ranz2}=\operatorname{sqr}\left(-2^{*} \log (\operatorname{ran} \times 1)\right)^{*} \sin \left(2^{*} \mathrm{pi}^{*} \operatorname{ran} \times 2\right) ;
\end{aligned}
$$

- Rangen is the uniform distribution random number generator

- Ranx1 and ranx2 are the uniformly distributed pair

- Ranz1 and ranz2 are the normally distributed pair

\title{
X. Simulation Flow Chart and Representative Results
}

\begin{abstract}
A flow chart of the complete simulation is shown in Figure 9. The data base contains the inputs to the simulation, and can be edited on-line before launching the simulation or off-line. The Monte-Carlo driver loops around the entire simulation. Outputs include a time plot of one of the runs (100 Monte Carlo runs were usually used) and a summary of results of the Monte Carlo runs.

An early simulation result is shown in Figure 10, no mixture ratio control on the left and with mixture ratio control on the right.
\end{abstract}

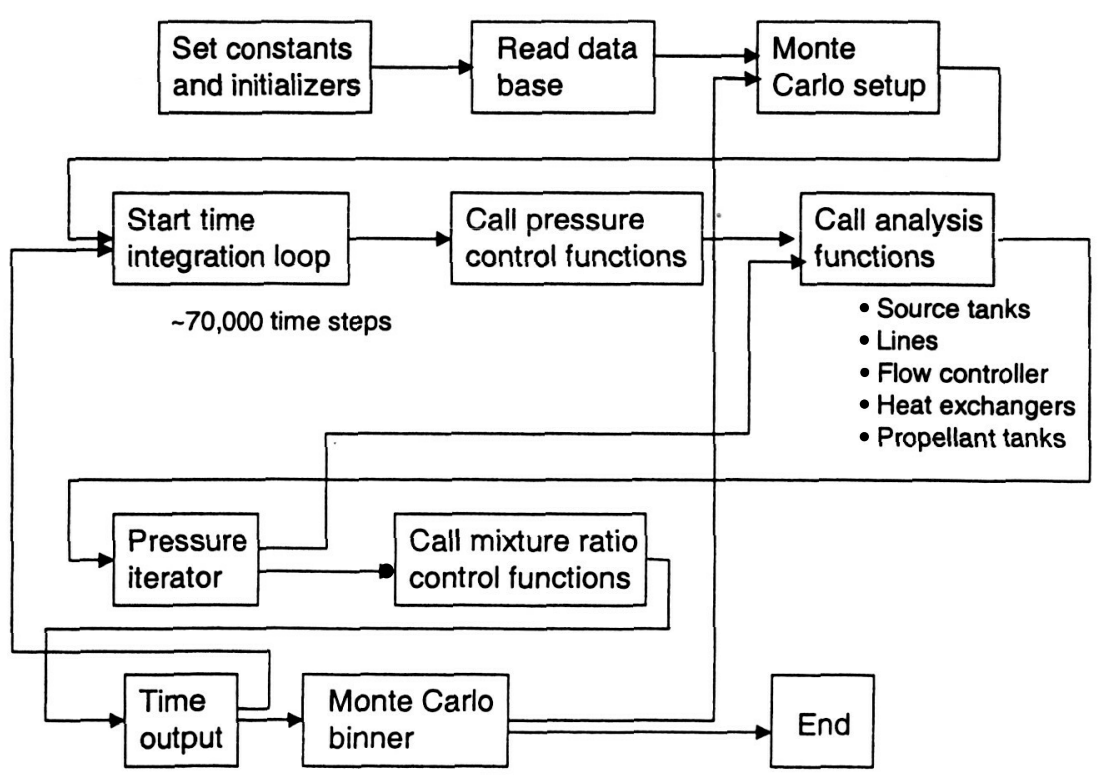

Figure 9: Simulation Logic Flow

The figure represents one time through the simulation. Without mixture ratio control, the ullage pressure controller maintains constant ullage pressure. The run mixture ratio is constant. Because the run mixture ratio is higher than the initially loaded mixture ratio, the mixture ratio remaining in the tanks decreases until oxidizer is depleted at tank mixture ratio zero. This case was a poorly calibrated system that had significant residuals in the no-control case. The curves on the right are with a PD controller (no integrator), perfect knowledge of initially loaded propellant, and perfect flowmeters. The mixture ratio controller alters ullage pressures, attempting to control the run mixture ratio to the initially loaded mixture ratio. Because the controller does not include an integrator, an error remains and gradually becomes larger. Tank pressures are adjusted by the controller until the pressures reach pre-set limits, and are then 
constant. Even without an integrator in the controller, the residual propellant is reduced by $75 \%$. An integrator was also tested, and in this case of perfect knowledge of tank liquid quantities and measurements, residual propellant decreased to near zero.

Type 1, tank quantities known and flows continuously measured, no integrator in controller. When integrator was added; residuals decreased further.
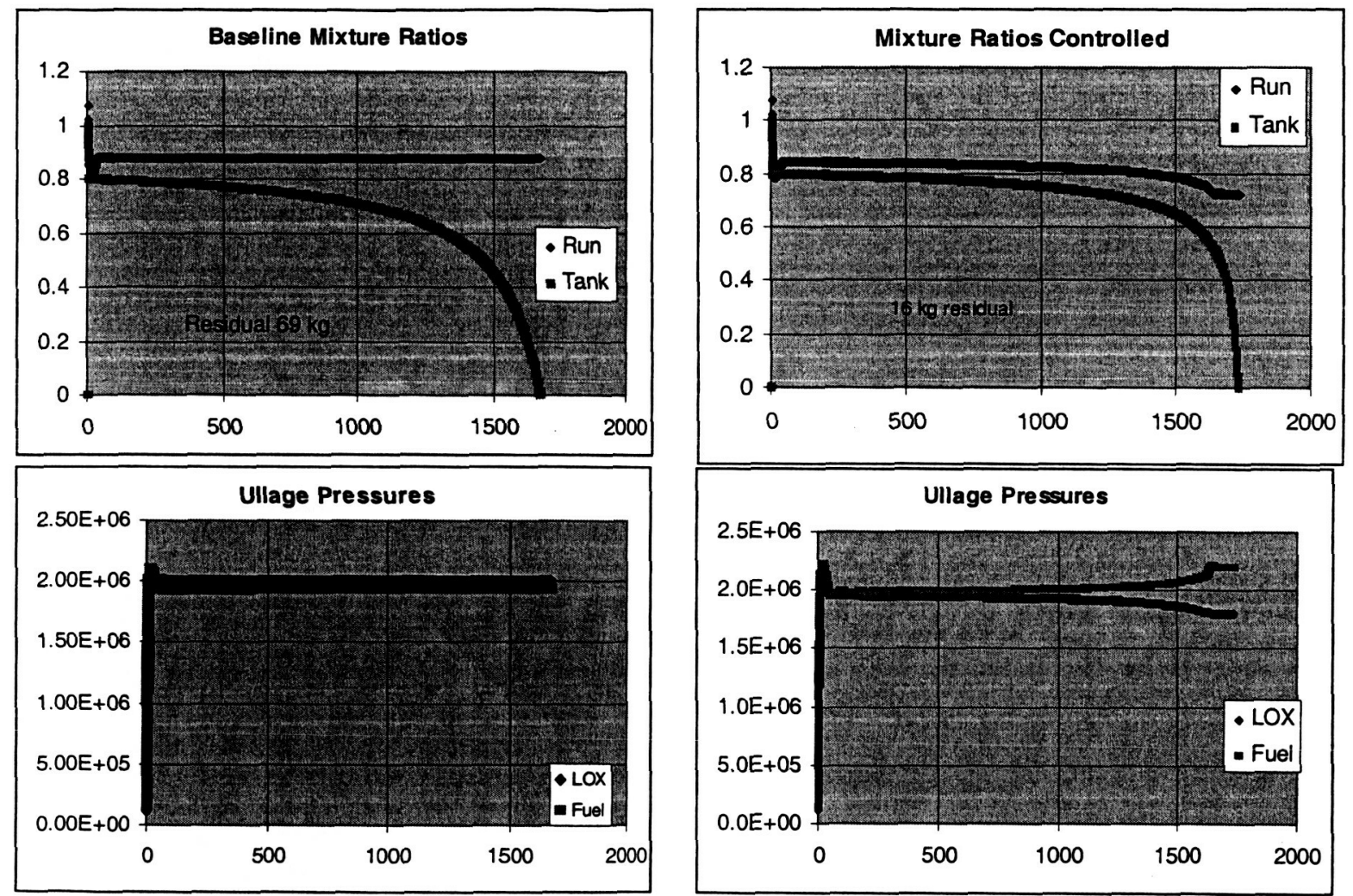

Figure 10: Early Simulation Run Results Summary

\section{Acknowledgement}

The work described in this paper was funded by the In-Space Propulsion Technology Program, managed by NASA's Science Mission Directorate in Washington, D.C., and implemented by the InSpace Propulsion Technology Office at Marshall Space Flight Center in Huntsville, Alabama. The program objective is to develop in-space propulsion technologies that can enable or benefit near and midterm NASA space science missions by significantly reducing cost, mass or travel times

\section{References}

1. The balanced flowmeter is a pressure-drop device being developed by the NASA Marshall Space Flight Center with A+FlowTek in Houston (www.aplusflowtek.com).

2. The PVT method is being developed by the NASA Marshall Space Flight Center with A+FlowTek in Houston (www.aplusflowtek.com). It is reportedly an improvement on earlier PVT methods.

3. The optical liquid quantity measurement device is being developed by the NASA Marshall Space Flight Center with a contractor.

4. http://encyclopedia.laborlawtalk.com/Box-Muller_transform 